\title{
NONLINEAR STABILITY OF VORTEX PATCHES
}

\author{
YUN TANG
}

\begin{abstract}
To establish the nonlinear (Liapunov) stability of both circular and elliptical vortex patches in the plane for the nonlinear dynamical system generated by the two-dimensional Euler equations of incompressible, inviscid hydrodynamics. This is accomplished by using a relative variational principle in terms of energy function. A counterexample shows that our result in the case of an elliptical vortex patch is the best one that can be attained by applying the energy estimate.
\end{abstract}

1. Introduction. The chief purpose of this paper is to establish the nonlinear stability of circular and elliptical vortex patches in the plane for the nonlinear dynamical system generated by the two-dimensional Euler equations of incompressible, inviscid hydrodynamics on $\mathbf{R}^{2}$.

The linear stability of a circular vortex patch was established by Kelvin [9], and the linear stability of a rotating Kirchhoff elliptical vortex patch was proved by Love [11] (see also [10, pp. 230, 232]). Recently, it was noted by Deem and Zabusky [6] that the Kirchhoff vortex patch is just the $m=2$ case of an infinite number of families of noncircular shapes with $m$-fold symmetry, which can be regarded as bifurcation from the circular one.

Over the past few years, an interest has grown in finite regions of uniform vorticity. See, for example, [5, 7, 14, 15 and 18].

Arnold $[1,2]$ has presented a method for proving a nonlinear version of the classical Rayleigh inflection point criterion for linear stability of shear flows. Recently, Arnold geometric setting has been exploited by a number of authors such as $[8,4,12,13,16$ and 17]. Arnold [3, p. 335] asserted that a stationary flow of an ideal fluid is in fact a conditional critical point of the kinetic energy; if this point is a nondegenerate extremum, then the stationary flow is stable. Unfortunately, Arnold's method does not apply directly to determine the stability of a vortex patch due to the discontinuity in the vorticity for which the differential calculus ideas in Arnold are not suitable. On the other hand, Deem and Zabusky [6] numerically show that a steady vortex patch may develop a thin arm. To overcome the difficulty concerning the discontinuity in the vorticity, Wan and Pulvirenti [18] provide a reduction procedure by which they established the nonlinear stability of circular vortex patches in a disk. The idea originally developed by Arnold $[1,2]$ and recently extended by Wan and Pulvirenti [18] is important to the formulation and execution of our results.

Received by the editors January 23, 1985 and, in revised form, December 22, 1986.

1980 Mathematics Subject Classification (1985 Revision). Primary 76C05, 58F10; Secondary 35P15, $26 \mathrm{D} 99$. 
In this paper, we use the energy as Liapunov functional to establish the $L^{1}$-stability of both circular and elliptical vortex patches in the plane. Our result on the stability of circular vortex patches is established in the whole plane while that of [18] is in a bounded disk. Moreover, for an elliptical vortex patch, which is a nonstationary rotating patch, the $L^{1}$-stability of Liapunov type is relative to a disk centered at the origin (see \$2) due to restrictions on the energy estimates in this case. A counterexample shows that our result is the best one that can be attained by applying the energy estimate. This answers one of the questions proposed in [18].

In order to obtain the energy estimates in $L^{1}$-space, we make use of the fact that $L^{1}$-perturbations in vorticity imply $C^{1}$-perturbations in stream function, by which perturbation in vorticity may be reduced from $L^{1}$-space to the $C^{1}$-radial case. Then the spectral analysis of the second order variation of the energy allows us to establish the energy estimate.

In $\S 2$ of this paper we give some basic concepts and state our main results, the stability theorems for both circular and elliptical vortex patches. The proofs depend on some energy estimates stated also in this section. In $\S 3$, we describe the reduction of the energy inequality from a small $L^{1}$-perturbation to the $C^{1}$-radial case. In $\S 4$, a second order Taylor expansion of the energy function is given in the radial $C^{1}$-case. Then, using the spectral analysis, we establish the energy estimates in this case. Finally, in $\$ 5$, we complete the proof of the energy estimates and the stability theorems stated in $\S 2$. A counterexample shows that some restrictions must be introduced to establish the energy inequality for elliptical vortex patches.

Following [18], a somewhat stronger stability result for both circular and elliptical vortex patch distributions in the plane may be established.

ACKNOWLEDGMENTS. This paper is a part of my dissertation at the Department of Mathematics, State University of New York at Buffalo. The author is grateful to Professor Yieh-Hei Wan for his patient supervision and invaluable help.

2. Basic concepts and the main results. Consider the motion of an incompressible, inviscid flow with unit density in the plane $\mathbf{R}^{2}$. The velocity field $\mathbf{u}=(u, v)$ can be described by a stream function $\psi, \mathbf{u}=\left(\psi_{y},-\psi_{x}\right)$. Define the vorticity $\omega=v_{x}-u_{y}$. Then, at any time, the vorticity satisfies the vorticity equation

$$
\omega_{t}+u \omega_{x}+v \omega_{y}=0
$$

and the Poisson equation

$$
\Delta \psi=-\omega,\left.\quad \psi_{x}\right|_{\infty}=\left.\psi_{y}\right|_{\infty}=0 .
$$

Write $\mathbf{x}=(x, y) \in \mathbf{R}^{2}$ and $|\mathbf{x}|^{2}=x^{2}+y^{2}$. The stream function $\psi$ then may be written as the logarithmic potential

$$
\psi=G \omega=(2 \pi)^{-1} \int \omega\left(\mathbf{x}^{\prime}\right) \log \left|\mathbf{x}-\mathbf{x}^{\prime}\right|^{-1} d x^{\prime} d y^{\prime},
$$

where $G$ is the Green's function for $-\Delta$, and the integral is taken over the whole plane. 
Let $A \subset \mathbf{R}^{2}$ be a region. A vortex patch $\omega$ is defined in the form $\lambda \chi_{A}$, where $\lambda \in \mathbf{R}^{1}$ and the area of $A$ are called the strength and the size of the patch $A$, respectively, and where $\chi_{A}$ is the characteristic function of $A$.

Denote by $\varphi_{t}(\omega), t \geqslant 0$, the vorticity at time $t$, with initial vorticity $\omega$. By the conservation laws, the circulation $\int \omega$, the center of vorticity $\int \mathbf{x} \omega=\int(x, y) \omega$ and the angular momentum $Q(\omega)=\int|\mathbf{x}|^{2} \omega$ are independent of $t$. Moreover, one has $\varphi_{t}\left(\lambda \chi_{A}\right)$ $=\lambda \chi_{A_{t}}$, where $A_{t}$ has the same area as $A$. Sometimes we also need to use the product of inertia of $\omega, \int x y \omega$. For a vortex patch $\omega_{0}=\chi_{A_{0}}$, write

$$
\mathscr{M}_{0}\left(\omega_{0}\right)=\left\{\omega=\chi_{A} \mid \int \omega=\int \omega_{0}, \int \mathbf{x} \omega=\int \mathbf{x} \omega_{0}\right\}
$$

and

$$
\mathscr{M}_{1}\left(\omega_{0}\right)=\left\{\left.\omega \in \mathscr{M}_{0}\left(\omega_{0}\right)\left|\int\right| \mathbf{x}\right|^{2} \omega=\int|\mathbf{x}|^{2} \omega_{0}\right\} .
$$

Then the sets $\mathscr{M}_{0}\left(\omega_{0}\right)$ and $\mathscr{M}_{1}\left(\omega_{0}\right)$ may be imagined as "invariant manifolds", $\varphi_{t}\left(\omega_{0}\right) \in \mathscr{M}_{0}\left(\omega_{0}\right)$ or $\mathscr{M}_{1}\left(\omega_{0}\right), \forall t \geqslant 0$.

Now we introduce the energy function of $\omega$,

$$
E(\omega)=\frac{1}{2}\langle\omega, G \omega\rangle=(4 \pi)^{-1} \int \omega(\mathbf{x}) \omega\left(\mathbf{x}^{\prime}\right) \log \left|\mathbf{x}-\mathbf{x}^{\prime}\right|^{-1} d x d y d x^{\prime} d y^{\prime}
$$

Then energy is also conserved, i.e. the energy function $E$ of $\varphi_{t}(\omega)$ is independent of $t$,

$$
E\left(\varphi_{t}(\omega)\right)=E(\omega) \text { for every } t \geqslant 0 .
$$

A vortex patch $\chi_{A}$ is called stationary if $\varphi_{t}\left(\chi_{A}\right)=\chi_{A} \forall t \geqslant 0 . \chi_{A}$ is called rotating with angular velocity $\Omega$ if $\varphi_{t}\left(\chi_{A}\right)=\chi_{R_{\Omega_{A} A}} \forall t \geqslant 0$, where $R_{\theta}$ is a rotation through angle $\theta$. Sometimes both of them are called steady. The basic patches considered in this paper are the circular vortex patch $\omega_{0}=\chi_{B(R)}$ and the elliptical vortex patch $\omega_{e}=\chi_{S}$, where

$$
\begin{gathered}
B(R)=\left\{(x, y) \mid x^{2}+y^{2} \leqslant R^{2}\right\}, \quad R \geqslant 0, \\
S=\left\{(x, y) \mid x^{2} / a^{2}+y^{2} / b^{2} \leqslant 1\right\}, \quad a>b>0 .
\end{gathered}
$$

It is well known that $\omega_{0}$ is a stationary vortex patch while $\omega_{e}$ is a nonstationary rotating patch with the angular velocity $\Omega=a b /(a+b)^{2}$.

In this section we state the main results of this paper, that is, the stability theorems for both circular and elliptical vortex patches in the plane, for which the corresponding energy estimates are also stated here.

Denote by $\|\cdot\|$ the $L^{1}$-norm of function space on $\mathbf{R}^{2}$. The stability theorem for circular vortex patches in the plane is

THEOREM 1. For any $\eta>0$, there exists a $\delta>0$ such that if a vortex patch $\omega$ satisfies $\left\|\omega-\omega_{0}\right\|<\delta$ and has uniformly bounded angular momentum $\int|\mathbf{x}|^{2} \omega$, then

$$
\left\|\varphi_{t}(\omega)-\omega_{0}\right\|<\eta, \quad \forall t>0 .
$$


REMARKS 1 . In [18] the stability theorem for circular vortex patches is established in a disk, while ours is in the whole plane.

2. As in [18], one may use an angular momentum estimate to prove the stability theorem for circular vortex patches. However, we prefer using the following energy estimate $\left(E_{1}\right)$ to prove it.

For a vortex patch $\omega_{0}=\chi_{A_{0}}$ and $\varepsilon>0$, write

$$
\mathscr{N}_{\varepsilon}\left(\omega_{0}\right)=\left\{\omega=\chi_{A} \mid\left\|\omega-\omega_{0}\right\|<\varepsilon\right\} .
$$

Proposition 1. Let $\omega_{0}=\chi_{B(R)}$. Then there exist $a C_{1}>0$ and an $\varepsilon>0$ such that for every vortex patch $\omega_{1}=\chi_{A} \in \mathscr{N}_{\varepsilon}\left(\omega_{0}\right) \cap \mathscr{M}_{0}\left(\omega_{0}\right)$ the energy inequality $\left(E_{1}\right)$ holds:

$$
E\left(\omega_{0}\right)-E\left(\omega_{1}\right) \geqslant C_{1}\left\|\omega_{0}-\omega_{1}\right\|^{4} \text {. }
$$

In the geometric setting of Arnold, the energy estimate $\left(E_{1}\right)$ means that the energy $E(\omega)$ as a Liapunov function has a "nondegenerate" local maximum at $\omega_{0}$ on the “invariant manifold" $\mathscr{M}_{0}\left(\omega_{0}\right)$.

The stability problem of elliptical vortex patches is more complicated. Let the ellipse $S$ be contained in a disk $D$ centered at the origin. The elliptical vortex patch $\omega_{e}=\chi_{S}$ is said to be $L^{1}$-stable relative to the disk $D$ if for any $\eta>0$, there exists a $\delta>0$ such that, to each vortex patch $\omega=\lambda \chi_{A}$ and each $t \geqslant 0$,

$$
\left\|\varphi_{t}(\omega)-\varphi_{i}\left(\omega_{e}\right)\right\|<\eta \quad \text { for some } \bar{t}
$$

provided $\left\|\omega-\omega_{e}\right\|<\delta$ and the support of $\varphi_{t^{\prime}}(\omega) \subset D$ for all $t^{\prime} \in[0, t]$. The stability theorem for elliptical vortex patches is

THEOREM 2. For the elliptical vortex patch $\omega_{e}=\chi_{s}$ given by (2.7), let the ratio $\gamma$ of the major to the minor axes be less than $3, \gamma=a / b<3$. Then there exists a disk $D$ containing $S$ such that $\omega_{e}$ is $L^{1}$-stable relative to $D$.

REMARK 3. In the definition of the $L^{1}$-stability of the elliptical vortex patch $\omega_{e}$, the reason for the choice of $\bar{t}=\bar{t}(t)$ is that a small perturbation of $\omega_{e}$ may result in a big difference in their vortex patches, since the angular velocity of $\omega_{e}, \Omega=$ $a b /(a+b)^{2}$, depends on the major and minor axes.

4. The stability of elliptical vortex patches is relative to a disk due to the restrictions on the following energy estimate $\left(E_{2}\right)$ by which its $L^{1}$-stability is established. And it could not be established by using only angular momentum estimates.

5. Love [11] proved that for $\gamma=a / b<3$ required in Theorem 2, the motion is linear stable. For $\gamma>3$, it can be proved that the motion is unstable. So $\gamma=3$ is a bifurcation point.

Write

$$
\mathscr{M}_{2}\left(\omega_{e}\right)=\left\{\omega \in \mathscr{M}_{1}\left(\omega_{e}\right) \mid \int x y \omega=0\right\} .
$$

The following estimate $\left(E_{2}\right)$ means that restricting to the cross section of the “invariant manifolds" $\mathscr{M}_{1}\left(\omega_{e}\right), \mathscr{M}_{2}\left(\omega_{e}\right)$, defined by product of inertia, $E\left(\omega_{1}\right)$ has a nondegenerate "local" maximum at $\omega_{e}$ on $\mathscr{M}_{2}\left(\omega_{e}\right)$ if the support of $\omega_{1}$ is confined 
to the range $D$. Then $E\left(\omega_{1}\right)$ as a Liapunov function can be applied to prove Theorem 2.

Proposition 2. For the elliptical vortex patch $\omega_{e}=\chi_{s}$ given by (2.7), let $\gamma=a / b$ $<3$. Then there exist $C_{2}>0, \varepsilon>0$ and a disk $D$ containing $S$, such that

$$
E\left(\omega_{e}\right)-E\left(\omega_{1}\right) \geqslant C_{2}\left\|\omega_{e}-\omega_{1}\right\|^{2}
$$

for $\omega_{1} \in \mathscr{N}_{\varepsilon}\left(\omega_{e}\right) \cap \mathscr{M}_{2}\left(\omega_{e}\right)$.

REMARK 6. Let the disk $D$ required in Proposition 2 have the largest radius $\mu=\mu(\gamma)$ where $\gamma=a / b$, then $d \mu / d \gamma<0$ and the value of $\mu$ is

$$
\mu(3)=1.189 \leqslant \mu \leqslant \mu(1)=1.874 ;
$$

see $\S 5$. A counterexample in $\S 5$ shows that without the restriction of the "range value" $\mu$ the energy estimate $\left(E_{2}\right)$ may fail. So our result is the best one that can be attained by applying the energy estimate.

3. The reduction procedure. In this section we show that under some assumptions, the energy inequality in $L^{1}$-space can be reduced to a radial $C^{1}$-case. The key point is to use the fact that $L^{1}$-perturbations of vortex patches imply $C^{1}$-perturbations of the corresponding stream functions. Thus, for an $L^{1}$-perturbation of the steady vortex patch $\omega_{0}=\chi_{A}$, we may make use of the $C^{1}$-stream function to produce a $C^{1}$-perturbation of the vortex patch $\omega_{0}$ instead of the orginal $L^{1}$-perturbation.

Let the region $A$ have the smooth boundary $\partial A$ such that the vortex patch $\omega_{0}=\chi_{A}$ is stationary relative to a frame with angular velocity $\Omega$. Then for the stream function $G \omega_{0}$ of $\omega_{0}$ there is a relative stream function

$$
\psi=G \omega_{0}+\frac{1}{2} \Omega\left(x^{2}+y^{2}\right)+C,
$$

such that $\left.\psi\right|_{\partial A}=0$, where $C$ is a constant.

Take a system of the local coordinates in some neighborhood of the closed contour $\partial A$, which is a diffeomorphism $\mathbf{x}(\xi, \eta)=(x(\xi, \eta), y(\xi, \eta))$ from an annular neighborhood in the polar coordinates with radius $\xi$ and angular $\eta$ to $\mathbf{R}^{2}$ such that $\mathbf{x}\left(\xi_{0}, \eta\right), 0 \leqslant \eta \leqslant 2 \pi$, represents the closed contour $\partial A$ with $\mathbf{x}(\xi, 0)=\mathbf{x}(\xi, 2 \pi)$ and the Jacobian $J(\xi, \eta)=\partial(x, y) / \partial(\xi, \eta)>0$.

Let $0 \leqslant \Omega<1$. From the maximum principle, it readily follows that $\psi(\mathbf{x})>0$ for any $\mathbf{x} \in$ Int $A$ and $\left.(\partial \psi / \partial \xi)\right|_{\partial A}<0$. The set $\{\mathbf{x} \mid \psi(\mathbf{x}) \geqslant 0\}$ may have two components of which $A$ is the inside one.

Let $D$ be a disk containing $A$. For an $L^{1}$-perturbation $\omega_{1}=\chi_{A_{1}} \in \mathscr{M}_{0}\left(\omega_{0}\right)$ of $\omega_{0}$ with $A_{1} \subset D$, it is well known that the relative stream function $\psi_{1}=G \omega_{1}+$ $\frac{1}{2} \Omega\left(x^{2}+y^{2}\right)+C$ of $\omega_{1}$ is $C^{1}$-close to $\psi$ on $D$.

In general, for any $C^{1}$-function $\tilde{\psi}$ which is $C^{1}$-close to $\psi$ on $D$, the set $\{\mathbf{x} \mid \tilde{\psi}(\mathbf{x}) \geqslant$ $0\}$ may also have two components of which the inside one is denoted by $\tilde{A}$. The vortex patch $\tilde{\omega}=\chi_{\tilde{A}}$ determined by $\tilde{\psi}$ is then radially $C^{1}$-close to $\omega_{0}$.

For both circular and elliptical vortex patches, we will prove later in this section that for every $L^{1}$-perturbation $\omega_{1}$ of $\omega_{0}$, there exists a $C^{1}$-function $\tilde{\psi}$ which is $C^{1}$-close to $\psi_{1}$ on $D$ (hence $C^{1}$-close to $\psi$ on $D$ ) such that the vortex patch $\tilde{\omega}=\chi_{\tilde{A}}$ 
determined by $\tilde{\psi}$ satisfies the following equalities

$$
\int \tilde{\omega}=\int \omega_{1}
$$

and

$$
\left\langle\omega_{1}-\tilde{\omega}, G \omega_{1}\right\rangle=\left\langle\omega_{1}-\tilde{\omega}, \tilde{\psi}\right\rangle .
$$

Before verifying the existence of such a $\tilde{\omega}$, let us analyze the relation between $\omega_{1}$ and $\tilde{\omega}$ required by (3.2) and (3.3). We have

$$
\begin{aligned}
E(\tilde{\omega})-E\left(\omega_{1}\right) & =\left\langle\tilde{\omega}-\omega_{1}, G \omega_{1}\right\rangle+\frac{1}{2}\left\langle\tilde{\omega}-\omega_{1}, G\left(\tilde{\omega}-\omega_{1}\right)\right\rangle \\
& \geqslant\left\langle\tilde{\omega}-\omega_{1}, G \omega_{1}\right\rangle=\left\langle\tilde{\omega}-\omega_{1}, \tilde{\psi}\right\rangle .
\end{aligned}
$$

REMARK 1 . Here we make use of the inequality

$$
\left\langle\tilde{\omega}-\omega_{1}, G\left(\tilde{\omega}-\omega_{1}\right)\right\rangle \geqslant 0,
$$

which readily follows from the Green's identity and the mean value theorem.

Thus, the energy estimates in $L^{1}$-space is reduced to determine the estimates of the right-hand side of (3.3).

LEMMA 3.1. Let $D$ be a disk containing $A$ such that the relative stream function $\psi$ of $\omega_{0}=\chi_{A}$ is negative on $D \backslash A$. Suppose that for every $L^{1}$-perturbation $\omega_{1}=\chi_{A_{1}}$ of $\omega_{0}$ with $A_{1} \subset D$, there is a $C^{1}$-function $\tilde{\psi}$ near the relative stream function $\psi_{1}$ of $\omega_{1}$ such that the vortex patch $\tilde{\omega}=\chi_{\tilde{A}}$ determined by $\tilde{\psi}$ (i.e. $\tilde{A}$ is the inside component of $\{\mathbf{x} \mid \tilde{\psi}(\mathbf{x}) \geqslant 0\}$ ) satisfies (3.2) and (3.3). Then

$$
E(\tilde{\omega})-E\left(\omega_{1}\right) \geqslant C_{1}\left\|\tilde{\omega}-\omega_{1}\right\|^{2},
$$

where $C_{1}>0$ is a priori constant independent of $\omega_{1}$ and $\tilde{\omega}$.

Proof. From (3.4), it suffices to prove

$$
\left\langle\tilde{\omega}-\omega_{1}, \tilde{\psi}\right\rangle \geqslant C_{1}\left\|\tilde{\omega}-\omega_{1}\right\|^{2} .
$$

Take a system of the local coordinates $(\xi, \eta)$ near $\partial A$ as above. For $(\xi, \eta)$ near $\partial \tilde{A}$, let $\xi^{\prime}=-\tilde{\psi}(\xi, \eta)+\xi_{0}, \eta^{\prime}=\eta$. Then $\left(\xi^{\prime}, \eta^{\prime}\right)$ can be regarded as a system of the local coordinates near $\partial \tilde{A}$ such that $\left(\xi_{0}, \eta^{\prime}\right), 0 \leqslant \eta^{\prime} \leqslant 2 \pi$, represents the closed contour $\partial \tilde{A}$.

Choose a constant $h_{1}>0$ so that the area of the set

$$
U=\left\{\left(\xi^{\prime}, \eta^{\prime}\right) \mid \xi_{0}-h_{1} \leqslant \xi^{\prime} \leqslant \xi_{0}\right\}
$$

is equal to the area of the set $\tilde{A} \backslash A_{1}, \int_{U}=\int_{\tilde{A} \backslash A_{1}}$. Then $\int_{\tilde{A} \backslash A_{1}} \tilde{\psi} \geqslant \int_{U} \tilde{\psi}$. Since

$$
\left\langle\tilde{\omega}-\omega_{1}, \tilde{\psi}\right\rangle=\left\langle\chi_{\tilde{A}}-\chi_{A_{1}}, \tilde{\psi}\right\rangle=\int_{\tilde{A} \backslash A_{1}} \tilde{\psi}-\int_{A_{1} \backslash \tilde{A}} \tilde{\psi},
$$

and $\tilde{\psi} \leqslant 0$ on $A_{1} \backslash \tilde{A}$, one has

$$
\left\langle\tilde{\omega}-\omega_{1}, \tilde{\psi}\right\rangle \geqslant \int_{\tilde{A} \backslash A_{1}} \tilde{\psi} \geqslant \int_{U} \tilde{\psi} .
$$


Let $\bar{J}$ be the Jacobian of the transformation $(x, y) \mapsto\left(\xi^{\prime}, \eta^{\prime}\right)$ and $\bar{J}_{0}=\left.\bar{J}\right|_{\xi^{\prime}=\xi_{0}}$. Then

$$
\begin{aligned}
\int_{U} \tilde{\psi} & =\int_{0}^{2 \pi} \int_{\xi_{0}-h_{1}}^{\xi_{0}} \tilde{\psi} \bar{J} d \xi^{\prime} d \eta^{\prime}=-\frac{1}{2}\left(\left.\int_{0}^{2 \pi} \frac{\partial \tilde{\psi}}{\partial \xi}\right|_{\xi^{\prime}=\xi_{0}} \bar{J}_{0} d \eta^{\prime}\right) h_{1}^{2}+o\left(\left|h_{1}\right|^{2}\right) \\
& \rightarrow \alpha_{1} h_{1}^{2}+o\left(\left|h_{1}\right|^{2}\right),
\end{aligned}
$$

as $\tilde{\psi} \rightarrow \psi$, where

$$
\alpha_{1}=-\left.\frac{1}{2} \int_{0}^{2 \pi} \frac{\partial \psi}{\partial \xi}\right|_{\xi=\xi_{0}} J_{0} d \eta>0
$$

On the other hand,

$$
\begin{aligned}
\left\|\tilde{\omega}-\omega_{1}\right\| & =\int_{\tilde{A} \backslash A_{1}}+\int_{A_{1} \backslash \tilde{A}}=2 \int_{U} \\
& =2 \int_{0}^{2 \pi} \int_{\xi_{0}-h_{1}}^{\xi_{0}} \bar{J} d \xi^{\prime} d \eta^{\prime}=2 h_{1} \int_{0}^{2 \pi} \bar{J}_{0} d \eta^{\prime}+o\left(\left|h_{1}\right|\right) \\
& \rightarrow \alpha_{2} h_{1}+o\left(\left|h_{1}\right|\right),
\end{aligned}
$$

as $\tilde{\psi} \rightarrow \psi$, where $\alpha_{2}=2 \int_{0}^{2 \pi} J_{0} d \eta>0$. So

$$
\int_{U} \tilde{\psi} \rightarrow \alpha_{1} \alpha_{2}^{-2}\left\|\tilde{\omega}-\omega_{1}\right\|^{2}+o\left(\left\|\tilde{\omega}-\omega_{1}\right\|^{2}\right) \text {. }
$$

Combining with (3.8), we get (3.7).

Consider the circular vortex patch $\omega_{0}=\chi_{B(R)}$. Using the definition of the stream function $G \omega_{0}$ in $\S 2$, we have, in polar coordinates,

$$
G \omega_{0}(\mathbf{x})= \begin{cases}\frac{1}{4}\left(R^{2}-R^{2} \log R^{2}-r^{2}\right), & \text { if } r \leqslant R, \\ -\frac{1}{2} R^{2} \log r, & \text { if } r>R,\end{cases}
$$

where we use $(r, \theta)$ for $(\xi, \eta)$. So $J=r$. Let $C=-\left.G \omega_{0}\right|_{r=R}$; then the relative stream function of $\omega_{0}$ is

$$
\psi=G \omega_{0}+C= \begin{cases}\frac{1}{4}\left(R^{2}-r^{2}\right), & \text { if } r \geqslant R, \\ -\frac{1}{2} R^{2} \log \frac{r}{R}, & \text { if } r<R .\end{cases}
$$

Here $\Omega=0$. We find $B(R)=\{\mathbf{x} \mid \psi(\mathbf{x}) \geqslant 0\}$.

For an $L^{1}$-perturbation $\omega_{1}=\chi_{A_{1}}$ of $\omega_{0}$, there is a real number $\mu$ near $0 \in \mathbf{R}$ such that the region $\tilde{A}=\left\{\mathbf{x} \mid G \omega_{1}(\mathbf{x})+C+\mu \geqslant 0\right\}$ has the same area as $A_{1}$. Write $\tilde{\omega}=\chi_{\tilde{A}}$ and $\tilde{\psi}=G \omega_{1}+C+\mu$. Then (3.2) holds, $\int \tilde{\omega}=\int \omega_{1}$. And (3.3) follows from

$$
\left\langle\omega_{1}-\tilde{\omega}, \tilde{\psi}-G \omega_{1}\right\rangle=(C+\mu) \int\left(\omega_{1}-\tilde{\omega}\right)=0 .
$$

We have

Lemma 3.2. Let $\omega_{0}=\chi_{B(R)}$. Then there exists a $C_{0}>0$ such that for every $L^{1}$-perturbation $\omega_{1}=\chi_{A_{1}}$ of $\omega_{0}$, there is a vortex patch $\tilde{\omega}=\chi_{\tilde{A}}$ which is radially $C^{1}$-close to $\omega_{0}$ and which satisfies

$$
E(\tilde{\omega})-E\left(\omega_{1}\right) \geqslant C_{0}\left\|\tilde{\omega}-\omega_{1}\right\|^{2} .
$$


Proof. Define $\tilde{\omega}$ as above. Since the relative stream function $\psi$ of $\omega_{0}$ given by (3.9) is negative outside of $B(R)$, the domain required in Lemma 3.1 can be consider as the whole plane. The conclusion then follows from Lemma 3.1.

Now we consider the elliptical vortex patch $\omega_{e}=\chi_{S}$ given by (2.7). Introduce the elliptical coordinates

$$
\left\{\begin{array}{l}
x=c \cosh \xi \cos \eta, \\
y=c \sinh \xi \sin \eta,
\end{array} \text { or } \quad z=\cosh \zeta,\right.
$$

where $c=\left(a^{2}-b^{2}\right)^{1 / 2}, z=x+i y, \zeta=\xi+i \eta, \xi \geqslant 0$ and $0 \leqslant \eta \leqslant 2 \pi$. Then $J$ $=\frac{1}{2} c^{2}(\cosh 2 \xi-\cos 2 \eta)$. Let $\xi_{0}>0$ satisfy $\cosh \xi_{0}=a / c$ or $\sinh \xi_{0}=b / c$, then the ellipse $S$ can be expressed as $\xi \leqslant \xi_{0}$. By the definition, the stream function of $\omega_{e}$ is (see also [10, p. 232])

$$
G \omega_{e}= \begin{cases}-\left(b x^{2}+a y^{2}\right) / 2(a+b)+d_{1}, & \text { if } \xi<\xi_{0}, \\ -\frac{1}{2} a b \xi-\frac{1}{4} a b e^{-2 \xi} \cos 2 \eta+d_{2}, & \text { if } \xi \geqslant \xi_{0},\end{cases}
$$

where

$$
d_{1}=-\frac{1}{2} a b \log \frac{a+b}{2}+\frac{1}{4} a b, \quad d_{2}=\frac{1}{2} a b \log \frac{c}{2} .
$$

Let $\Omega=a b /(a+b)^{2}$ and $C=-\frac{1}{4} a b\left(2 \log 2(a+b)^{-1}+\left(a^{2}+b^{2}\right)(a+b)^{-2}\right)$. Then the relative stream function of $\omega_{0}$ is

$$
\begin{aligned}
\psi= & G \omega_{e}+\frac{1}{2} \Omega\left(x^{2}+y^{2}\right)+C \\
= & \left\{\begin{array}{l}
-\frac{1}{2} a^{2} b^{2}(a+b)^{-2}\left(a^{-2} x^{2}+b^{-2} y^{2}-1\right), \quad \text { if } \xi<\xi_{0}, \\
-\frac{1}{2} a b\left(\xi-\xi_{0}\right)-\frac{1}{4} a b\left(e^{-2 \xi}-e^{-2 \xi_{0}}\right) \cos 2 \eta \\
\\
\quad+\frac{1}{4} a b e^{-2 \xi_{0}}\left(\cosh 2 \xi-\cosh 2 \xi_{0}\right), \quad \text { if } \xi \geqslant \xi_{0} .
\end{array}\right.
\end{aligned}
$$

The set $\{\mathbf{x} \mid \psi(\mathbf{x}) \geqslant 0\}$ has two connected components of which $S$ is the inside one and the outside is unbounded.

Since $\Omega>0$, we cannot obtain a $\tilde{\omega}$ satisfying (3.3) by using the similar method as before. Furthermore, as we have seen in Proposition 2 that in order to obtain the energy estimate of the elliptical vortex patch $\omega_{e}=\chi_{s}$, a perturbation $\omega_{1}$ of $\omega_{e}$ must be in $\mathscr{M}_{2}\left(\omega_{e}\right)$. Thus we need the following special lemma to verify the conditions (3.2) and (3.3).

Lemma 3.3. Let $D$ be a disk containing the ellipse $S$. For an $L^{1}$-perturbation $\omega_{1}=\chi_{A_{1}} \in \mathscr{M}_{2}\left(\omega_{e}\right)$ of $\omega_{e}$ with $A_{1} \subset D$, there exists a $C^{1}$-function $\tilde{\psi}$ near the relative stream function $\psi$ of $\omega_{e}$ given by (3.12) on $D$ such that for the inside component $\tilde{A}$ of the set $\{\mathbf{x} \mid \tilde{\psi}(\mathbf{x}) \geqslant 0\}$, the vortex patch $\tilde{\omega}=\chi_{\tilde{A}} \in \mathscr{M}_{2}\left(\omega_{e}\right)$ satisfies

$$
\left\langle\tilde{\omega}-\omega_{1}, \tilde{\psi}-G \omega_{1}\right\rangle=0 .
$$

Proof. We use the Implicit Function Theorem (IFT) to find such a $\tilde{\psi}$.

Consider the $C^{1}$-function space $\mathscr{E}=\left\{f: D \rightarrow \mathbf{R} \mid f\right.$ are $\left.C^{1}\right\}$ with $C^{1}$-norm. For $\bar{\psi} \in \mathscr{E}$ near $\psi$ on $D$, let

$$
\psi_{\mu}=\bar{\psi}+\frac{1}{2} \mu_{1}\left(x^{2}+y^{2}\right)+\mu_{2} x y+\mu_{3} x+\mu_{4} y+\mu_{5},
$$


where $\mu=\left(\mu_{1}, \ldots, \mu_{5}\right) \in \mathbf{R}^{5}$. Then for $\mu$ close to $0 \in \mathbf{R}^{5}, \psi_{\mu}$ is $C^{1}$-close to $\psi$ on $D$. Denote by $A_{\mu}$ the inside component of the set $\left\{\mathbf{x} \in D \mid \psi_{\mu}(\mathbf{x}) \geqslant 0\right\}$ and $\omega_{\mu}=\chi_{A_{\mu}}$. Define

$$
F=\left(F_{1}, \ldots, F_{5}\right): \mathscr{E} \times \mathbf{R}^{5} \rightarrow \mathbf{R}^{5}
$$

near $(\psi, 0) \in \mathscr{E} \times \mathbf{R}^{5}$ as follows:

$$
\begin{aligned}
& F_{1}(\bar{\psi}, \mu)=\int|\mathbf{x}|^{2}\left(\omega_{\mu}-\omega_{e}\right), \quad F_{2}(\bar{\psi}, \mu)=\int x y \omega_{\mu}, \\
& F_{3}(\bar{\psi}, \mu)=\int x \omega_{\mu}, \quad F_{4}(\bar{\psi}, \mu)=\int y \omega_{\mu}, \quad F_{5}(\bar{\psi}, \mu)=\int\left(\omega_{\mu}-\omega_{e}\right) .
\end{aligned}
$$

Then $F(\psi, 0)=0$.

Since $\partial A_{\mu}$ is radially $C^{1}$-close to $\partial S, A_{\mu}$ can be described by $A_{\mu}=\left\{(\xi, \eta) \mid \xi \leqslant \xi_{0}\right.$ $+h\}$, where $h=h(\cdot ; \bar{\psi}, \mu): S^{1} \rightarrow \mathbf{R}$ is a $C^{1}$-function on the unit circle $S^{1}$ and $h$ satisfies

$$
\psi_{\mu}\left(\xi_{0}+h, \eta\right)=0 .
$$

Then $h(\eta ; \psi, 0)=0$. The continuities of $\partial F_{i} / \partial \mu_{j}, i, j=1, \ldots, 5$, near $(\bar{\psi}, \mu)=$ $(\psi, 0)$, readily follow from $\psi_{\mu} \in C^{1}$.

Let us calculate $\partial F_{i} / \partial \mu_{j}$ at $(\psi, 0)$. It is not hard to check,

$$
\frac{\partial \psi_{\mu}}{\partial h}=\left.\frac{\partial \psi}{\partial \xi}\right|_{\xi=\xi_{0}}=a b(a+b)^{-2}\left(a^{2} \sin ^{2} \eta+b^{2} \cos ^{2} \eta\right)
$$

at $(\bar{\psi}, \mu)=(\psi, 0)$. By $(3.15)$,

$$
\frac{\partial h}{\partial \mu_{i}}=\frac{\partial \psi_{\mu} / \partial \mu_{i}}{\partial \psi_{\mu} / \partial h}
$$

Note $F_{1}(\psi, 0)=\int_{0}^{2 \pi} \int_{\xi_{0}}^{\xi_{0}+h}|\mathbf{x}|^{2} J d \xi d \eta$, where $J$ is the Jacobian from $(x, y)$ to $(\xi, \eta)$. One has, at $(\psi, 0)$,

$$
\begin{aligned}
\frac{\partial F_{1}}{\partial \mu_{1}} & =\left.\int_{0}^{2 \pi}|\mathbf{x}|^{2} J\right|_{\xi=\xi_{0}} \frac{\partial h}{\partial \mu_{1}} d \eta \\
& =\frac{(a+b)^{2} c^{4}}{8 a b} \int_{0}^{2 \pi}\left(\cos 2 \xi_{0}+\cos 2 \eta\right)^{2} d \eta \\
& =\frac{(a+b)^{2}}{8 a b}\left(a^{2}+b^{2}\right)\left(3 a^{2}+b^{2}\right) \pi
\end{aligned}
$$

Similar computations of $\partial F_{i} / \partial \mu_{j}, i, j=1, \ldots, 5$, yield

$$
\operatorname{det}\left(\frac{\partial F_{i}}{\partial \mu_{j}}\right)=\frac{1}{4 a b} c^{2}(a+b)^{10}\left(a^{2}+b^{2}\right)^{3}>0 .
$$

Then by the IFT, for every $C^{1}$-perturbation $\bar{\psi}$ of $\psi$, there exists a unique $\mu \in \mathbf{R}^{5}$ near 0 such that $F(\bar{\psi}, \mu)=0$.

Now for an $L^{1}$-perturbation $\omega_{1} \in \mathscr{M}_{2}\left(\omega_{e}\right)$ of $\omega_{e}$, let

$$
\bar{\psi}=G \omega_{1}+\frac{1}{2} \Omega\left(x^{2}+y^{2}\right)+C
$$


and $\tilde{\psi}=\psi_{\mu}$ given by (3.14). Using the definition of $F$, we find that $\tilde{\omega}=\omega_{\mu} \in$ $\mathscr{M}_{2}\left(\omega_{e}\right)$. Since

$$
\tilde{\psi}-G \omega_{1}=\frac{1}{2}\left(\Omega+\mu_{1}\right)\left(x^{2}+y^{2}\right)+\mu_{2} x y+\mu_{3} x+\mu_{4} y+\mu_{5}+C,
$$

and $\omega_{1} \in \mathscr{M}_{2}\left(\omega_{e}\right)$, (3.13) then holds.

Thus, for an $L^{1}$-perturbation $\omega_{1} \in \mathscr{M}_{2}\left(\omega_{e}\right)$ of $\omega_{e}$, we can find a vortex patch $\tilde{\omega}$ which is radially $C^{1}$-close to $\omega_{1}$. That means the energy inequality in $L^{1}$-space can be reduced to a radial $C^{1}$-case.

Lemma 3.4. Let $D$ be a disk containing the ellipse $S$ such that the relative stream function $\psi$ of $\omega_{e}=\chi_{S}$ is negative on $D \backslash S$. Then there exists a constant $C_{1}>0$ such that for any $L^{1}$-perturbation $\omega_{1}=\chi_{A} \in \mathscr{M}_{2}\left(\omega_{e}\right)$ of $\omega_{e}$ with $A_{1} \subset D$, one has a vortex patch $\tilde{\omega} \in \mathscr{M}_{2}\left(\omega_{e}\right)$ which is radially $C^{1}$-close to $\omega_{1}$ and satisfies

$$
E(\tilde{\omega})-E\left(\omega_{1}\right) \geqslant C_{1}\left\|\tilde{\omega}-\omega_{1}\right\|^{2}
$$

Proof. Choose $\tilde{\omega}$ as in Lemma 3.3. Then $\tilde{\omega}$ satisfies (3.2) and (3.3). The conclusion follows from Lemma 3.1.

4. The energy estimates for the $C^{1}$-radial case. In this section we first give the second order Taylor expansion of the energy $E\left(\omega_{0}\right)$. Then through the spectral analysis of both circular and elliptical vortex patches for the second order operator of the energy, we establish their energy estimates for the $C^{1}$-radial case.

For a rotating vortex patch $\omega_{0}=\chi_{A}$ and its relative stream function $\psi$ given in (3.1), take a system of the local coordinates near $\partial A$ as in complex form

$$
x(\xi, \eta)=x(\xi, \eta)+i y(\xi, \eta), \quad z(\xi, 0)=z(\xi, 2 \pi),
$$

so that $z\left(\xi_{0}, \eta\right), 0 \leqslant \eta \leqslant 2 \pi$, represents the closed contour $\partial A$ and the Jacobian $J(\xi, \eta)=\partial(x, y) / \partial(\xi, \eta)>0$.

Let us consider a $C^{1}$-perturbation $\tilde{z}=\tilde{x}+i \tilde{y}$ of $z\left(\xi_{0}, \eta\right)$ in the radial case, that is

$$
\tilde{z}=\tilde{z}(\eta)=z\left(\xi_{0}+h(\eta), \eta\right), \quad 0 \leqslant \eta \leqslant 2 \pi,
$$

where $h \in C^{1}\left(S^{1}, \mathbf{R}\right)$ with $|h|_{\infty}$ small. Denote by $A_{h}$ the region of $\mathbf{R}^{2}$ enclosed by $\tilde{z}(\eta)$. Write $\omega_{h}=\chi_{A_{h}}$. Then $\omega_{0}=\chi_{A_{0}}=\chi_{A}$. Let

$$
J_{0}(\eta)=J\left(\xi_{0}, \eta\right), \quad q=J_{0} h \quad \text { and } \quad|h|_{2}^{2}=\int_{0}^{2 \pi} h^{2}(\eta) d \eta .
$$

For a $C^{1}$-function $f(z)$ defined near $\partial A$, one has

$$
\begin{aligned}
\left\langle\omega_{h}-\omega_{0}, f\right\rangle & =\int_{0}^{2 \pi} \int_{\xi_{0}}^{\xi_{0}+h} f(z(\xi, \eta)) J(\xi, \eta) d \xi d \eta \\
& =\int_{0}^{2 \pi} f_{0} q d \eta+o\left(|h|_{2}\right),
\end{aligned}
$$

where

$$
f_{0}(\eta)=f\left(z\left(\xi_{0}, \eta\right)\right)
$$


Write

$$
\begin{array}{r}
\mathscr{T}_{0}\left(\omega_{0}\right)=\left\{h \in C^{1}\left(S^{1}, \mathbf{R}\right) \mid \int\left(\omega_{h}-\omega_{0}\right)=0, \int z\left(\omega_{h}-\omega_{0}\right)=0\right. \\
\text { and } \left.h(s)+\xi_{0} \geqslant \varepsilon>0, \forall s \in S^{1}\right\},
\end{array}
$$

and

$$
\mathscr{T}_{1}\left(\omega_{0}\right)=\left\{\left.h \in \mathscr{T}_{0}\left(\omega_{0}\right)\left|\int\right| z\right|^{2}\left(\omega_{h}-\omega_{0}\right)=0\right\}
$$

$\mathscr{T}_{0}$ and $\mathscr{T}_{1}$ may be imagined as "tangent spaces" to the "invariant manifolds" $\mathscr{M}_{0}$ and $\mathscr{M}_{1}$ at $\omega_{0}$, respectively.

The following lemma gives the second order Taylor expansion of the energy $E(\omega)$.

LEMMA 4.1. Let $\omega_{0}=\chi_{A}$ be a nonstationary rotating vertex patch with the smooth boundary $\partial A$. Then for a $C^{1}$-perturbation $h \in \mathscr{T}_{1}\left(\omega_{0}\right)$ of $\partial A$, the energy $E\left(\omega_{h}\right)$ satisfies

$$
E\left(\omega_{h}\right)-E\left(\omega_{0}\right)=\frac{1}{2}\langle q, \mathscr{L} q\rangle+o\left(|h|_{2}^{2}\right)
$$

where

$$
\begin{aligned}
q & =J_{0} h, \quad \mathscr{L}(q)=I_{0} q+\int_{0}^{2 \pi} K\left(\eta, \eta^{\prime}\right) q\left(\eta^{\prime}\right) d \eta^{\prime} \\
I_{0} & =\left.\left(\frac{\partial \psi / \partial \xi}{J}\right)\right|_{\xi=\xi_{0}}, \quad K\left(\eta, \eta^{\prime}\right)=\frac{1}{2 \pi} \log r_{0}^{-1} \text { and } \\
r_{0} & =\left|z\left(\xi_{0}, \eta\right)-z\left(\xi_{0}, \eta^{\prime}\right)\right| .
\end{aligned}
$$

Furthermore, if $\omega_{0}=\chi_{A}$ is a stationary vortex patch, then for a $C^{1}$-perturbation $h \in \mathscr{T}_{0}\left(\omega_{0}\right)$ of $\partial A,(4.3)$ also holds.

Proof. It is easy to see

$$
E\left(\omega_{h}\right)-E\left(\omega_{0}\right)=\left\langle\omega_{h}-\omega_{0}, G \omega_{0}\right\rangle+\frac{1}{2}\left\langle\omega_{h}-\omega_{0}, G\left(\omega_{h}-\omega_{0}\right)\right\rangle
$$

For the relative stream function $\psi=G \omega_{0}+\frac{1}{2} \Omega\left(x^{2}+y^{2}\right)+C$. Since $h \in \mathscr{T}_{0}\left(\omega_{0}\right)$ for $\Omega=0$ and $h \in \mathscr{T}_{1}\left(\omega_{0}\right)$ for $\Omega \neq 0$, we have

$$
\left\langle\omega_{h}-\omega_{0}, G \omega_{0}\right\rangle=\left\langle\omega_{h}-\omega_{0}, \psi\right\rangle=\frac{1}{2}\left\langle I_{0} q, q\right\rangle+o\left(|h|_{2}^{2}\right)
$$

Thus, it suffices to show

$$
\left\langle\omega_{h}-\omega_{0}, G\left(\omega_{h}-\omega_{0}\right)\right\rangle=\int_{0}^{2 \pi} \int_{0}^{2 \pi} K\left(\eta, \eta^{\prime}\right) q\left(\eta^{\prime}\right) q(\eta) d \eta d \eta^{\prime}+o\left(|h|_{2}^{2}\right)
$$


Let $r=\left|z(\xi, \eta)-z\left(\xi^{\prime}, \eta^{\prime}\right)\right|, J^{\prime}=J\left(\xi^{\prime}, \eta^{\prime}\right), q^{\prime}=q\left(\eta^{\prime}\right)$ and $h^{\prime}=h\left(\eta^{\prime}\right)$. One has

$$
\begin{aligned}
&\left|\left\langle\omega_{h}-\omega_{0}, G\left(\omega_{h}-\omega_{0}\right)\right\rangle-\int_{0}^{2 \pi} \int_{0}^{2 \pi} K q q^{\prime} d \eta d \eta^{\prime}\right| \\
&\left.=\frac{1}{2 \pi}\left|\int_{0 \leqslant \eta, \eta^{\prime} \leqslant 2 \pi}\right| \int_{\xi_{0}}^{\xi_{0}+h^{\prime}} \int_{\xi_{0}}^{\xi_{0}+h} J J^{\prime} \log r^{-1} d \xi d \xi^{\prime}-q q^{\prime} \log r_{0}^{-1}\right] \mid \\
& \leqslant \frac{1}{2 \pi} \int_{\left|\eta-\eta^{\prime}\right| \geqslant \delta}\left|\int_{\xi_{0}}^{\xi_{0}+h} \int_{\xi_{0}}^{\xi_{0}+h^{\prime}} J J^{\prime} \log r^{-1} d \xi d \xi^{\prime}-q q^{\prime} \log r_{0}^{-1}\right| \\
&+\frac{1}{2 \pi} \int_{\left|\eta-\eta^{\prime}\right|<\delta}\left|\int_{\xi_{0}}^{\xi_{0}+h} \int_{\xi_{0}}^{\xi_{0}+h^{\prime}} J J^{\prime} \log r^{-1} d \xi d \xi^{\prime}\right| \\
&+\frac{1}{2 \pi} \int_{\left|\eta-\eta^{\prime}\right|<\delta}\left|q q^{\prime} \log r_{0}^{-1}\right|, \text { for } \delta>0 \text { small. }
\end{aligned}
$$

It is not hard to check that for any $\varepsilon>0$, there is a $\delta>0$ such that

$$
\begin{aligned}
& \int_{\left|\eta-\eta^{\prime}\right|<\delta}\left|\log r_{0}^{-1}\right|<\varepsilon \text { and } \\
& \int_{\left|\eta-\eta^{\prime}\right|<\delta} \int_{\xi_{0}}^{\xi_{0}+h} \int_{\xi_{0}}^{\xi_{0}+h^{\prime}}\left|\log r^{-1}\right| d \xi d \xi^{\prime} d \eta d \eta^{\prime}<\varepsilon .
\end{aligned}
$$

Applying the Mean Value Theorem and the inequality $\left|h h^{\prime}\right| \leqslant \frac{1}{2}\left(|h|^{2}+\left|h^{\prime}\right|^{2}\right)$, we find that the right-hand side of (4.5) is

$$
\leqslant \varepsilon|h|_{2}^{2}+M_{1} \varepsilon|h|_{2}^{2}+M_{2} \varepsilon|h|_{2}^{2} \leqslant \varepsilon M|h|_{2}^{2} .
$$

So (4.4) holds.

From Lemma 4.1 we can see that the energy inequalities for the $C^{1}$-radial case are valid so long as the operator $\mathscr{L}$ of the quadratic term of $E(\omega)$ remains negative definite. That is

Lemma 4.2. Let the operator $\mathscr{L}$ given in Lemma 4.1 be negative definite, i.e. there is a $C_{0}>0$ such that

$$
\langle q, \mathscr{L} q\rangle<-C_{0}|h|_{2}^{2}
$$

for $q=J_{0} h$. Then there is $C_{1}>0$ independent of $h$ such that

$$
E\left(\omega_{0}\right)-E\left(\omega_{h}\right) \geqslant C_{1}\left\|\omega_{h}-\omega_{0}\right\|^{2} .
$$

Proof. By Lemma 4.1,

$$
E\left(\omega_{0}\right)-E\left(\omega_{h}\right)=-\frac{1}{2}\langle q, \mathscr{L} q\rangle+o\left(|h|_{2}^{2}\right)>C_{0}^{\prime}|h|_{2}^{2} .
$$

On the other hand,

$$
\left\|\omega_{h}-\omega_{0}\right\|=\int_{0}^{2 \pi}\left|\int_{\xi_{0}}^{\xi_{0}+h} J d \xi\right| d \eta \leqslant \int_{0}^{2 \pi} J_{0}|h| d \eta+C^{\prime}|h|_{2}^{2} .
$$

By the Schwarz inequality, $\left\|\omega_{h}-\omega_{0}\right\| \leqslant C_{1}^{\prime}|h|_{2}$. So (4.7) holds. 
For the circular vortex patch $\omega_{0}=\chi_{B(R)}$ and its relative stream function given by (3.9), we have $J=r, q=R h$ and $I_{0}=-\frac{1}{2}$. So

$$
\mathscr{L} q=-\frac{1}{2} q+\int_{0}^{2 \pi} K\left(\theta, \theta^{\prime}\right) q\left(\theta^{\prime}\right) \theta^{\prime}
$$

where

$$
K\left(\theta, \theta^{\prime}\right)=-\frac{1}{2 \pi} \log \left(R\left|1-e^{i\left(\theta^{\prime}-\theta\right)}\right|\right) .
$$

The following lemma verifies the energy estimate of circular vortex patches for the $C^{1}$-radical case.

Lemma 4.3. Let $\omega_{0}=\chi_{B(R)}$. Then there exists a $C_{0}>0$ such that for every $C^{1}$-perturbation $\omega_{h}$ of $\omega_{0}$ with $h \in \mathscr{T}_{0}\left(\omega_{0}\right)$, one has

$$
E\left(\omega_{0}\right)-E\left(\omega_{h}\right) \geqslant C_{0}\left\|\omega_{0}-\omega_{h}\right\|^{2} .
$$

Proof. By Lemma 4.2, it is suffices to prove the negative definite property of the operator $\mathscr{L}$ given by (4.8).

Set $\bar{\theta}=\theta^{\prime}-\theta$. One has $\mathscr{L}\left(e^{i n \theta}\right)=\alpha_{n} e^{i n \theta}$, where

$$
\alpha_{n}=-\frac{1}{2}-\frac{1}{2 \pi} \int_{0}^{2 \pi} \log \left(R\left|1-e^{i \bar{\theta}}\right|\right) e^{i n \bar{\theta}} d \bar{\theta} .
$$

So $e^{i n \theta}, n=0,1, \ldots$, are eigenfunctions of $\mathscr{L}$ with the eigenvalues $\alpha_{n}$. By computation, $\alpha_{n}=\frac{1}{2}(1 / n-1)$ for $n>0$. Thus $\alpha_{n} \leqslant \alpha_{2}=-\frac{1}{4}<0$ for $n \geqslant 2$.

On the other hand, for the Fourier expansion of $q=R h$,

$$
q=\sum q_{n} e^{i n \theta}, \quad q_{n}=\frac{1}{2 \pi} \int_{0}^{2 \pi} q e^{i n \theta} d \theta .
$$

Since $h \in \mathscr{T}_{0}$, using (4.2), one has

$$
\begin{aligned}
& 0=\int_{0}^{2 \pi} \int_{R}^{R+h} r d r=2 \pi q_{0}+o\left(|h|_{2}\right) \text { and } \\
& 0=\int_{0}^{2 \pi}\left[(R+h)^{2}-R^{2}\right] e^{i \theta} d \theta=4 \pi q_{1}+o\left(|h|_{2}\right) .
\end{aligned}
$$

So $q_{0}=o\left(|h|_{2}\right)$ and $q_{1}=o\left(|h|_{2}\right)$. Therefore

$$
\langle q, \mathscr{L} q\rangle=\sum_{n=0}^{\infty}\left|q_{n}\right|^{2} \alpha_{n}<-\frac{1}{4} \sum_{n=2}^{\infty}\left|q_{n}\right|^{2}+o\left(|h|_{2}^{2}\right)<-C_{1}|h|_{2}^{2} .
$$

For the elliptical vortex patch $\omega_{e}=\chi_{s}$, as we have seen in Proposition 2, the ratio $\gamma$ of the major to the minor axes is required to be less than 3 , and its perturbation must remain in the "cross section" $\mathscr{M}_{2}\left(\omega_{e}\right)$. Write

$$
\mathscr{T}_{2}\left(\omega_{e}\right)=\left\{h \in \mathscr{T}_{1}\left(\omega_{e}\right) \mid \int x y \omega_{h}=\int x y \omega_{e}=0\right\} .
$$

Then $\mathscr{T}_{2}\left(\omega_{e}\right)$ may be regarded as the "tangent set" of $\mathscr{M}_{2}\left(\omega_{e}\right)$. Following the above approach, we have the energy estimate of $\omega_{e}=\chi_{s}$ for the $C^{1}$-radial case. 
Lemma 4.4. For the elliptical vortex patch $\omega_{e}=\chi_{s}$, let $\gamma=a / b<3$. Then there exists a constant $C_{2}>0$ such that for every $C^{1}$-perturbation $\omega_{h}$ of $\omega_{e}$ with $h \in \mathscr{T}_{2}\left(\omega_{e}\right)$, one has

$$
E\left(\omega_{e}\right)-E\left(\omega_{h}\right) \geqslant C_{2}\left\|\omega_{e}-\omega_{h}\right\|^{2}
$$

Proof. Using the elliptical coordinates $z=c \cosh \zeta$ given by (3.11) and the relative stream function $\psi$ of $\omega_{e}$ given by (3.12), we find that

$$
I_{0}=\left.\left(\frac{\partial \psi / \partial \xi}{J}\right)\right|_{\xi=\xi_{0}}=-\frac{a b}{(a+b)^{2}}
$$

and

$$
\mathscr{L} q=-a b(a+b)^{-2} q+\int_{0}^{2 \pi} K\left(\eta, \eta^{\prime}\right) q\left(\eta^{\prime}\right) d \eta^{\prime}
$$

where

$$
K\left(\eta, \eta^{\prime}\right)=-\frac{1}{2 \pi} \log \left(c\left|\cosh \left(\xi_{0}+i \eta\right)-\cosh \left(\xi_{0}+i \eta^{\prime}\right)\right|\right) .
$$

By Lemma 4.2, it suffices to verify the negative definite property of the operator $\mathscr{L}$.

Since

$$
\left|\cosh \left(\xi_{0}+i \eta\right)-\cosh \left(\xi_{0}+i \eta^{\prime}\right)\right|=\frac{1}{2} e^{\xi_{0}}\left|\left(1-e^{-i \eta+i \eta^{\prime}}\right)\left(1-e^{-2 \xi_{0}-i \eta-i \eta^{\prime}}\right)\right|,
$$

one has

$$
\begin{aligned}
\mathscr{L} e^{i m \eta}= & -a b(a+b)^{-2} e^{i m \eta}+\frac{1}{2 \pi}\left(\xi_{0}-\log \frac{c}{2}\right) \int_{0}^{2 \pi} e^{i m \eta^{\prime}} d \eta^{\prime} \\
& -\frac{1}{2} I_{m}(-i \eta)-\frac{1}{2} I_{m}\left(2 \xi_{0}+i \eta\right),
\end{aligned}
$$

where

$$
I_{m}(\alpha+i \beta)=\frac{1}{\pi} \int_{0}^{2 \pi}\left(\log \left|1-e^{\alpha+i \beta+i \eta}\right|\right) e^{i m \eta} d \eta .
$$

Let $\alpha_{0}=\mathscr{L} e^{0}$. Computations yield that for $m>0$,

$$
\begin{aligned}
\mathscr{L} e^{i m \eta} & =-a b(a+b)^{-2} e^{i m \eta}+\frac{1}{m} e^{-m \xi_{0}} \cosh \left(m \xi_{0}+i m \eta\right) \\
& =\alpha_{m} \cos m \eta+i \beta_{m} \sin m \eta,
\end{aligned}
$$

where

$$
\begin{aligned}
& \alpha_{m}=-a b(a+b)^{-2}+\frac{1}{m} e^{-m \xi_{0}} \cosh m \xi_{0}, \\
& \beta_{m}=-a b(a+b)^{-2}+\frac{1}{m} e^{-m \xi_{0}} \sinh m \xi_{0} .
\end{aligned}
$$

So $\alpha_{m}$ and $\beta_{m}$ are eigenvalues of the linear operator $\mathscr{L}$ with the associated eigenfunctions $\cos m \eta$ and $\sin m \eta$, respectively. 
Note $\gamma=a / b<3$. It readily follows that for $m \geqslant 3$, the eigenvalues

$$
\begin{aligned}
\beta_{m} & \leqslant \alpha_{m} \leqslant \alpha_{3}=-a b(a+b)^{-2}+\frac{1}{3} e^{-3 \xi_{0}} \cosh 3 \xi_{0} \\
& =\gamma^{2}(\gamma-3) / 3(1+\gamma)^{3}=-C^{\prime}<0 .
\end{aligned}
$$

Consider the Fourier expansion of $q=J_{0} h$,

$$
q=\frac{1}{2} a_{0}+\sum_{m=1}^{\infty}\left(a_{m} \cos m \eta+b_{m} \sin m \eta\right)
$$

where

$$
a_{m}=\frac{1}{\pi} \int_{0}^{2 \pi} q \cos m \eta d \eta, \quad b_{m}=\frac{1}{\pi} \int_{0}^{2 \pi} q \sin m \eta d \eta .
$$

One has

$$
\begin{aligned}
\langle q, \mathscr{L} q\rangle & =\frac{1}{4} a_{0}^{2} \alpha_{0}+\sum_{m=1}^{\infty}\left(\alpha_{m} a_{m}^{2}+\beta_{m} b_{m}^{2}\right) \\
& \leqslant-C^{\prime} \sum_{m=3}^{\infty}\left(a_{m}^{2}+b_{m}^{2}\right)+\frac{1}{4} a_{0}^{2} \alpha_{0}+\sum_{l=1}^{2}\left(\alpha_{l} a_{l}^{2}+\beta_{l} b_{l}^{2}\right),
\end{aligned}
$$

and $|q|_{2}^{2}=a_{0}^{2}+\sum_{m=1}^{\infty}\left(a_{m}^{2}+b_{m}^{2}\right)$. Thus, it suffices to show that the first $m<3$ terms of the expansion are of higher order than $|h|_{2}^{2}$, i.e.

$$
a_{0}, a_{1}, a_{2}, b_{1}, b_{2}=o\left(|h|_{2}\right) .
$$

Let us make use of a general equality. For any $C^{1}$-function $f$ defined near $\partial A$ with $\int\left(\omega_{h}-\omega_{e}\right) f=0,(4.2)$ implies

$$
\int_{0}^{2 \pi} f_{0} q d \eta=o\left(|h|_{2}\right), \quad f_{0}=\left.f\right|_{\xi=\xi_{0}} .
$$

Since $h \in \mathscr{T}_{2}\left(\omega_{e}\right)$, we can identify the function $f$ with $1, z,|z|^{2}$ or $x y$ to get (4.12) as follows.

For $f=f_{0}=1,(4.13)$ becomes

$$
\int_{0}^{2 \pi} q d \eta=\pi a_{0}=o\left(|h|_{2}\right), \quad \text { or } a_{0}=o\left(|h|_{2}\right) ;
$$

for $f=z$ and $f_{0}=c \cosh \left(\xi_{0}+i \eta\right),(4.13)$ becomes

$$
c \int_{0}^{2 \pi} q \cosh \left(\xi_{0}+i \eta\right) d \eta=a \pi a_{1}+i b \pi b_{1}=o\left(|h|_{2}\right),
$$

or $a_{1}, a_{2}=o\left(|h|_{2}\right)$; for $f=|z|^{2}$ and $f_{0}=\left|z_{0}\right|^{2}=\frac{1}{2} c^{2}\left(\cosh 2 \xi_{0}+\cos 2 \eta\right)$, (4.13) becomes

$$
\frac{1}{2} \pi c^{2}\left(a_{0} \cosh 2 \xi_{0}+a_{2}\right)=o\left(|h|_{2}\right),
$$

or, by (4.14), $a_{2}=o\left(|h|_{2}\right)$; for $f=x y$ and $f_{0}=x_{0} y_{0}=\frac{1}{2} a b \sin 2 \eta$, (4.13) becomes $\frac{1}{2} a b b_{2}=o\left(|h|_{2}\right)$ or $b_{2}=o\left(|h|_{2}\right)$. Thus, (4.12) holds.

5. Proofs of the main results and a discussion. Now we use the reduction procedure in $\S 3$ and the energy estimates for the $C^{1}$-radial case in $\S 4$ to prove the propositions and the theorems stated in $\S 2$. 
Moreover, for elliptical vortex patches, we discuss in this section the restriction of the energy estimate to a bounded disk which, as we have seen in $\$ 2$, influences the result on the $L^{1}$-stability.

In order to complete the proof of the stability theorems, we should employ another energy inequality and a vortex inequality.

LEMmA 5.1. There is a constant $C_{0}>0$ such that for vortex patches $\omega_{0}=\chi_{A_{0}}$ and $\omega_{1}=\chi_{A_{1}}$ if $\omega_{1}$ is $L^{1}$-close to $\omega_{0}$ and $A_{0}$ and $A_{1}$ are uniformly bounded (i.e. there is $M>0$ such that $\left.A_{0}, A_{1} \subset B(M)\right)$, then

$$
E\left(\omega_{0}\right)-E\left(\omega_{1}\right) \leqslant C_{0}\left\|\omega_{0}-\omega_{1}\right\| .
$$

Proof. From

$$
E\left(\omega_{0}\right)-E\left(\omega_{1}\right)=\frac{1}{2}\left\langle\omega_{0}-\omega_{1}, G \omega_{1}\right\rangle-\frac{1}{2}\left\langle\omega_{0}, G\left(\omega_{1}-\omega_{0}\right)\right\rangle,
$$

Using the boundedness of $G$ on $B(M)$, one has

$$
\begin{aligned}
E\left(\omega_{0}\right)-E\left(\omega_{1}\right) & \leqslant \frac{1}{2} \sup _{B(M)}\left|G \omega_{1}\right|\left\|\omega_{0}-\omega_{1}\right\|+\frac{1}{2}|G|_{B(M)}\left\|\omega_{1}-\omega_{0}\right\| \\
& \leqslant C_{0}\left\|\omega_{1}-\omega_{0}\right\| .
\end{aligned}
$$

LEMMA 5.2. Let $\omega_{1}=\chi_{A}$ have zero center of vorticity, i.e. $\int \mathbf{x} \omega_{1}=0$. Then there is $C_{1}>0$ such that for every vortex patch $\omega=\chi_{B}$ with uniformly bounded angular momentum, $Q(\omega) \leqslant M$, one has

$$
\left\|\varphi_{t}(\tilde{\omega})-\varphi_{t}(\omega)\right\| \leqslant C_{1}\left\|\omega-\omega_{1}\right\|^{1 / 2}
$$

where $\tilde{\omega}$ is given by

$$
\tilde{\omega}(\mathbf{x})=\omega(\mathbf{x}+\tilde{\mathbf{x}}) \text { and } \tilde{\mathbf{x}}=\left(\int \omega\right)^{-1} \int \mathbf{x} \omega .
$$

Proof. It is not hard to check that there is $C_{1}^{\prime}>0$ such that

$$
\left\|\varphi_{t}(\tilde{\omega})-\varphi_{t}(\omega)\right\| \leqslant C_{1}^{\prime}|\tilde{\mathbf{x}}| .
$$

On the other hand, from $\tilde{\mathbf{x}}=\left(\int \omega\right)^{-1} \int \mathbf{x}\left(\omega-\omega_{1}\right)$ and the Schwarz inequality, one has

$$
\begin{aligned}
|\tilde{\mathbf{x}}|^{2} & \leqslant\left(\int \omega\right)^{-2}\left(\int|\mathbf{x}|^{2}\left|\omega-\omega_{1}\right|\right)\left\|\omega-\omega_{1}\right\| \\
& \leqslant\left(\int \omega\right)^{-2}\left(Q(\omega)+Q\left(\omega_{1}\right)\right)\left\|\omega-\omega_{1}\right\| .
\end{aligned}
$$

So (5.2) holds.

Let us now consider the circular vortex patch $\omega_{0}=\chi_{B(R)}$.

Proof of Proposition 1. By Lemma 3.2, there is $C_{1}^{\prime}>0$ such that for any $L^{2}$-perturbation $\omega_{1} \in \mathscr{M}_{0}\left(\omega_{0}\right)$, there is $\bar{\omega}$ radially $C^{1}$-ciose to $\omega_{0}$ satisfying $\int \bar{\omega}=\int \omega_{1}$ and

$$
E(\bar{\omega})-E\left(\omega_{1}\right) \geqslant C_{1}^{\prime}\left\|\bar{\omega}-\omega_{1}\right\|^{2} .
$$

By Lemma 4.3, there is $C_{2}^{\prime}>0$ such that for a $C^{1}$-perturbation $\tilde{\omega} \in \mathscr{M}_{0}\left(\omega_{0}\right)$, one has

$$
E\left(\omega_{0}\right)-E(\tilde{\omega}) \geqslant C_{2}^{\prime}\left\|\tilde{\omega}-\omega_{0}\right\|^{2} .
$$


Let $\tilde{\omega}$ be given by $\tilde{\omega}(\mathbf{x})=\bar{\omega}(\mathbf{x}+\tilde{\mathbf{x}})$, where $\tilde{\mathbf{x}}=\left(\int \omega\right)^{-1} \int \mathbf{x} \bar{\omega}$. One has $E(\bar{\omega})=E(\tilde{\omega})$. So

$$
E\left(\omega_{0}\right)-E\left(\omega_{1}\right) \geqslant C_{1}^{\prime}\left\|\bar{\omega}-\omega_{1}\right\|^{2}+C_{2}^{\prime}\left\|\tilde{\omega}-\omega_{0}\right\|^{2} .
$$

Using (5.2) (at $t=0$ ), one has $\left\|\tilde{\omega}-\omega_{1}\right\| \geqslant C_{3}^{\prime}\|\bar{\omega}-\tilde{\omega}\|^{2}$. So (5.4) becomes

$$
\begin{aligned}
E\left(\omega_{0}\right)-E\left(\omega_{1}\right) & \geqslant \frac{1}{2} C_{1}^{\prime}\left\|\bar{\omega}-\omega_{1}\right\|^{2}+C_{4}^{\prime}\|\bar{\omega}-\tilde{\omega}\|^{4}+C_{2}^{\prime}\left\|\tilde{\omega}-\omega_{0}\right\|^{2} \\
& \geqslant C_{5}^{\prime}\left(\left\|\omega_{1}-\bar{\omega}\right\|^{4}+\|\bar{\omega}-\tilde{\omega}\|^{4}+\left\|\tilde{\omega}-\omega_{0}\right\|^{4}\right) \geqslant C_{1}\left\|\omega_{1}-\omega_{0}\right\|^{4} .
\end{aligned}
$$

Proof of TheOREM 1. For the circular vortex patch $\omega_{0}=\chi_{B(R)}$, it suffices to show that there exist $C^{\prime}>0$ and $\beta>0$ such that

$$
\left\|\varphi_{t}(\omega)-\omega_{0}\right\| \leqslant C^{\prime}\left\|\omega-\omega_{0}\right\|^{\beta} .
$$

For a vortex patch $\omega=\lambda \chi_{A} L^{1}$-close to $\omega_{0}$, define $\tilde{\omega}$ and $\omega^{*}$ by $\tilde{\omega}(\mathbf{x})=\omega(\mathbf{x}+\tilde{\mathbf{x}})$, $\tilde{\mathbf{x}}=\left(\int \omega\right)^{-1} \int \mathbf{x} \omega$, and $\omega^{*}=\lambda \chi_{B\left(R^{\prime}\right)}, \int \omega^{*}=\int \omega$. Then

$$
\left\|\varphi_{t}(\omega)-\omega_{0}\right\| \leqslant\left\|\varphi_{t}(\omega)-\varphi_{t}(\tilde{\omega})\right\|+\left\|\varphi_{t}(\tilde{\omega})-\omega^{*}\right\|+\left\|\omega^{*}-\omega_{0}\right\| .
$$

We now estimate the terms of the right-hand side of (5.6). For the first term, by Lemma 5.2, we have

$$
\left\|\varphi_{t}(\tilde{\omega})-\varphi_{t}(\omega)\right\| \leqslant C_{1}^{\prime}\left\|\omega-\omega_{0}\right\|^{1 / 2} \text {. }
$$

For the second term, it is easy to see that the inequality $\left(E_{1}\right)$ is valid also for $\omega^{*}$ and $\varphi_{t}(\tilde{\omega})$. Therefore

$$
\begin{aligned}
\left\|\varphi_{t}(\tilde{\omega})-\omega^{*}\right\| & \leqslant C_{1}\left|E\left(\varphi_{t}(\tilde{\omega})\right)-E\left(\omega^{*}\right)\right|^{1 / 4} \\
& =C_{1}\left|E(\tilde{\omega})-E\left(\omega^{*}\right)\right|^{1 / 4} \\
& \leqslant C_{2}\left\|\tilde{\omega}-\omega^{*}\right\|^{1 / 4} \quad \text { (by Lemma 5.1). }
\end{aligned}
$$

Using Lemma 5.2, one readily has

$$
\left\|\tilde{\omega}-\omega^{*}\right\| \leqslant\|\tilde{\omega}-\omega\|+\left\|\omega-\omega^{*}\right\| \leqslant C_{3}^{\prime}\left\|\omega-\omega_{0}\right\|^{1 / 2} .
$$

Thus

$$
\left\|\varphi_{t}(\tilde{\omega})-\omega^{*}\right\| \leqslant C_{3}\left\|\omega-\omega_{0}\right\|^{1 / 8} .
$$

For the third term, it is easy to check

$$
\left\|\omega^{*}-\omega_{0}\right\| \leqslant C_{4}\left\|\omega-\omega_{0}\right\| \text {. }
$$

From (5.6)-(5.9), we have $\left\|\varphi_{t}(\omega)-\omega_{0}\right\| \leqslant C^{\prime}\left\|\omega-\omega_{0}\right\|^{1 / 8}$, which is (5.5).

For the elliptical patch $\omega_{e}=\chi_{S}$, let $D$ be a disk containing $S$ such that the relative stream function $\psi$ given by (3.12) is positive inside of $S$ and negative on $D \backslash S$. The proof of the corresponding energy estimate $\left(E_{2}\right)$ is

Proof of Proposition 2. For $\omega_{e}=\chi_{s}$, by Lemma 3.4 and Lemma 4.4, there are constants $C_{1}^{\prime}$ and $C_{2}^{\prime}$ such that for an $L^{1}$-perturbation $\omega_{1}=\chi_{A} \in \mathscr{M}_{2}\left(\omega_{e}\right)$ of $\omega_{e}$ with $A \subset D$, corresponding to a vortex patch $\tilde{\omega} \in \mathscr{M}_{2}\left(\omega_{e}\right)$ radially $C^{1}$-close to $\omega_{e}$, one has

$$
E(\tilde{\omega})-E\left(\omega_{1}\right) \geqslant C_{1}^{\prime}\left\|\tilde{\omega}-\omega_{1}\right\|^{2}
$$


and

Then

$$
E\left(\omega_{e}\right)-E(\tilde{\omega}) \geqslant C_{2}^{\prime}\left\|\omega_{e}-\tilde{\omega}\right\|^{2}
$$

$$
\begin{aligned}
E\left(\omega_{e}\right)-E\left(\omega_{1}\right) & \geqslant C_{1}^{\prime}\left\|\tilde{\omega}-\omega_{1}\right\|^{2}+C_{2}^{\prime}\left\|\omega_{e}-\tilde{\omega}\right\|^{2} \\
& \geqslant C_{3}^{\prime}\left(\left\|\tilde{\omega}-\omega_{1}\right\|+\left\|\omega_{e}-\tilde{\omega}\right\|\right)^{2} \geqslant C_{2}\left\|\omega_{e}-\omega_{1}\right\|^{2} .
\end{aligned}
$$

To establish the stability of elliptical vortex patches, we need the following lemma, which can be regarded as a corollary of Proposition 2.

LeMma 5.3. For $\omega_{e}=\chi_{S}$ and $\varepsilon>0$, there is $\delta>0$ such that for an $L^{1}$-perturbation $\tilde{\omega} \in \mathscr{M}_{2}\left(\omega_{e}\right) \cap \mathscr{N}_{\delta}\left(\omega_{e}\right)$ of $\omega_{e}$, to each $t \geqslant 0$, one has

$$
\left\|\varphi_{t}(\tilde{\omega})-\varphi_{t_{1}}\left(\omega_{e}\right)\right\|<\varepsilon
$$

for some $t_{1}$ provided $\varphi_{t^{\prime}}(\tilde{\omega}) \subset D \forall t^{\prime} \in[0, t]$.

Proof. Since the motion of $\omega_{e}, \varphi_{t}\left(\omega_{e}\right)$ is uniformly rotating, by evaluation, there are $\tau \neq 0$ and $\alpha \neq 0$ such that

$$
\int x y \varphi_{s}\left(\omega_{e}\right)=\alpha \sin \frac{2 \pi}{\tau} s, \quad \forall s \geqslant 0 .
$$

Let $I(s, \omega)=\int x y \varphi_{\tau+s}(\omega) . I(s, \omega)$ is clearly continuous at $\left(0, \omega_{e}\right)$ in an appropriate space. From (5.11), one has

$$
I\left(0, \omega_{e}\right)=0 \text { and } \partial I\left(0, \omega_{e}\right) / \partial s=2 \pi \alpha / \tau \neq 0 .
$$

By the IFT, there is a unique number $s=s(\omega)$ near $0 \in \mathbf{R}$ such that

$$
I(s(\omega), \omega)=\int x y \varphi_{\tau+s(\omega)}(\omega)=0 .
$$

So $P(\omega)=\varphi_{\tau+s(\omega)}(\omega)$ is a Poincaré map of the closed orbit $\left\{\varphi_{t}\left(\omega_{e}\right) \mid 0 \leqslant t \leqslant \tau\right\}$.

Consider a neighborhood $U$ of $\omega_{e}$ in $\mathscr{M}_{2}\left(\omega_{e}\right)$ such that for every $\omega \in U$, the energy estimate $\left(E_{2}\right)$

$$
E\left(\omega_{e}\right)-E(\omega) \geqslant C_{1}\left\|\omega-\omega_{e}\right\|^{2}
$$

holds and the Poincare map $P(\omega)$ is well defined. The following claim shows the stability of $P(\omega)$.

Claim. For any $\eta>0$ there is a neighborhood of $\omega_{e}$ which is contained in the $\eta$-ball

$$
B_{\eta} \subset B\left(\omega_{e}, \eta\right)=\left\{\omega \in U \mid\left\|\omega_{e}-\omega\right\| \leqslant \eta\right\}
$$

and which is invariant under $P(\omega), P\left(B_{\eta}\right) \subset B_{\eta}$. In fact, let

$$
B_{\eta}=\left\{\omega \in U \mid E(\omega) \geqslant E\left(\omega_{e}\right)-C_{1} \eta^{2}\right\} .
$$

It easily follows from (5.12) that $B_{\eta} \subset B\left(\omega_{e}, \eta\right)$. One can take $\eta>0$ sufficiently small so that $P\left(B_{\eta}\right) \subset U$. Then for $\omega \in B_{\eta}$, by the conservation law of energy, one has

$$
E(P(\omega))=E\left(\varphi_{\tau+s(\omega)}(\omega)\right)=E(\omega) \geqslant E\left(\omega_{e}\right)-C_{1} \eta^{2} .
$$

So $P(\omega) \in B_{\eta}$. 
For each $t^{\prime} \in[0, t]$, there are a nonegative integer $N$ and $\bar{t} \in[0, \tau+1]$ such that $\varphi_{t^{\prime}}(\tilde{\omega})=\varphi_{i}\left(P^{N}(\tilde{\omega})\right)$. Using the stability of the Poincare map $P$ at $\omega_{e}$, and the compactness of $[0, \tau+1]$, there are $t_{1}$ close to $\bar{t}$ and $\delta>0$ such that

$$
\left\|\varphi_{t^{\prime}}(\tilde{\omega})-\varphi_{t_{1}}\left(\omega_{e}\right)\right\|<\varepsilon
$$

for $\tilde{\omega} L^{1}$-close to $\omega_{e},\left\|\tilde{\omega}-\omega_{e}\right\|<\delta$.

Proof of Theorem 2. For $\omega_{e}=\chi_{s}$ and any $\eta>0$ we want to find $\delta>0$ so that for a vortex patch $\omega=\lambda \chi_{A}$ and $t \geqslant 0$ with $\left\|\omega-\omega_{e}\right\|<\delta$ and $\varphi_{t^{\prime}}(\omega) \subset D, \forall t^{\prime} \in$ $[0, t]$, one has

$$
\left\|\varphi_{t}(\omega)-\varphi_{i}\left(\omega_{e}\right)\right\|<\eta \quad \text { for some } \bar{t} .
$$

We divide the left-hand side of (5.13) into several terms.

Let $\tilde{\omega}(\mathbf{x})=\omega(\mathbf{x}+\tilde{\mathbf{x}})$ with $\tilde{\mathbf{x}}=\left(\int \omega\right)^{-1} \int \mathbf{x} \omega$. By Lemma 5.2, there is $\delta_{1}>0$ such that

$$
\left\|\varphi_{t}(\omega)-\varphi_{t}(\tilde{\omega})\right\|<\eta / 3 \text { for }\left\|\omega-\omega_{e}\right\|<\delta_{1} .
$$

Let $\omega_{e}=\lambda \chi_{\bar{s}}$, where

$$
\bar{S}=\left\{(x, y) \mid \frac{x^{2}}{\bar{a}^{2}}+\frac{y^{2}}{\bar{b}^{2}} \leqslant 1\right\} \text { with } \int \chi_{\bar{S}}=\int \chi_{A} \text { and } \int|\mathbf{x}|^{2} \omega_{e}=\int|\mathbf{x}|^{2} \tilde{\omega} .
$$

By Lemma 5.3, there is $\delta_{2}>0$ such that for $\left\|\omega-\omega_{e}\right\|<\delta_{2}$, one has $t_{1}$ and

$$
\left\|\varphi_{t}(\tilde{\omega})-\varphi_{t_{1}}\left(\omega_{e}\right)\right\|<\eta / 3 \text {. }
$$

We can choose $\bar{t}>0$ so that $\varphi_{t_{1}}\left(\omega_{\bar{e}}\right)$ and $\varphi_{\bar{t}}\left(\omega_{e}\right)$ have overlapping major and minor axes. It is not hard to check that there is $\delta_{3}>0$ such that

$$
\left\|\varphi_{t_{1}}\left(\omega_{\bar{e}}\right)-\varphi_{\bar{t}}\left(\omega_{e}\right)\right\|<\eta / 3 \text { for }\left\|\omega-\omega_{e}\right\|<\delta_{3} .
$$

Then for $\delta=\min \left\{\delta_{1}, \delta_{2}, \delta_{3}\right\}$ and $\left\|\omega-\omega_{e}\right\|<\delta$, one has

$$
\begin{aligned}
\left\|\varphi_{t}(\omega)-\varphi_{i}\left(\omega_{e}\right)\right\| \leqslant & \left\|\varphi_{t}(\omega)-\varphi_{t}(\tilde{\omega})\right\|+\left\|\varphi_{t}(\tilde{\omega})-\varphi_{t_{1}}\left(\omega_{\bar{e}}\right)\right\| \\
& +\left\|\varphi_{t_{1}}\left(\omega_{\bar{e}}\right)-\varphi_{i}\left(\omega_{e}\right)\right\|<\eta . \quad \square
\end{aligned}
$$

For the elliptical vortex patch $\omega_{e}=\chi_{S}$, we find that both the $L^{1}$-stability and the energy estimate $\left(E_{2}\right)$ are relative to a bounded disk $D$ which contains the ellipse $S$ so that the relative stream function $\psi$ of $\omega_{e}$ is negative on $D \backslash S$. Let $R_{0}$ be the largest radius $R$ such that the disk $D=B(R)$ with the above property. Let the ratio $\gamma=a / b \in(1,3)$ and $R_{0}=\mu a$, where $\mu=\mu(\gamma)$. By calculation, we find $d \mu / d \gamma<0$ and

$$
\mu(1)=1.874 \geqslant \mu \geqslant \mu(3)=1.189 .
$$

The following counterexample explains that the restriction of the "range value" for the energy estimate $\left(E_{2}\right)$ cannot be dispensed with. 
EXAMPLE. We construct a vortex patch $\omega=\chi_{A} \in \mathscr{M}_{2}\left(\omega_{e}\right)$ so that

$$
E(\omega)-E\left(\omega_{e}\right)>0,
$$

i.e. the energy estimate $\left(E_{2}\right)$ does not hold.

By (3.5),

$$
\begin{aligned}
E(\omega)-E\left(\omega_{e}\right) & =\left\langle\omega-\omega_{e}, G \omega_{e}\right\rangle+\frac{1}{2}\left\langle\omega-\omega_{e}, G\left(\omega-\omega_{e}\right)\right\rangle \\
& \geqslant\left\langle\omega-\omega_{e}, G \omega_{e}\right\rangle .
\end{aligned}
$$

Claim. There is an $R>0$ such that

$$
\psi(\mathbf{x}) \geqslant \frac{1}{3} \Omega r^{2} \quad \text { for } r=|\mathbf{x}| \geqslant R,
$$

where $\Omega=a b /(a+b)^{2}$. In fact, for $\xi>\xi_{0}$, using (3.12), one has

$$
\begin{aligned}
\frac{\partial}{\partial \xi}\left(\psi-\frac{1}{3} \Omega r^{2}\right) & =\frac{\partial}{\partial \xi}\left(G \omega_{e}+\frac{1}{6} \Omega r^{2}\right) \\
& =\frac{1}{2} a b+\frac{1}{2} a b e^{-2 \xi} \cos 2 \eta+\frac{1}{6} c^{2} \sinh 2 \xi>0,
\end{aligned}
$$

for $\xi$ large enough. So (5.16) holds for $R$ large enough.

For $\varepsilon>0$ and $0<\alpha<1$, take symmetry regions $A_{1}, A_{2}$ and $B_{0}$ such that

(i) $A_{1}$ (or $B_{0}$ ) is a pair of small disks connecting the ends of the minor (major) axes in the exterior (interior) of the ellipse $S$, and $A_{2}$ is an annular of radius $R$, as in Figure 1.

(ii) Their areas are $\int_{B_{0}}=\varepsilon, \int_{A_{1}}=(1-\alpha) \varepsilon$ and $\int_{A_{2}}=\alpha \varepsilon$.

(iii) Their angular momentum satisfy $\int_{A_{1}} r^{2}+\int_{A_{2}} r^{2}=\int_{B_{0}} r^{2}$.

Let $A=\left(S \backslash B_{0}\right) \cup A_{1} \cup A_{2}$ and $\omega=\chi_{A}$. Then $\omega \in \mathscr{M}_{2}\left(\omega_{e}\right)$. Since $\int_{B_{0}} r^{2}=a^{2} \varepsilon$ $+o(\varepsilon), \int_{A_{1}} r^{2}=b^{2}(1-\alpha) \varepsilon+o(\varepsilon)$ and $\int_{A_{2}} r^{2}=R^{2} \alpha \varepsilon+o(\varepsilon)$. By (iii), one has $R^{2} \alpha \varepsilon$ $=a^{2} \varepsilon-b^{2}(1-\alpha) \varepsilon+o(\varepsilon)$, or $R^{2}=\left[a^{2}-b^{2}(1-\alpha)\right] / \alpha+o(1)$. So $R^{2} \rightarrow+\infty$ as $\alpha \rightarrow 0$.

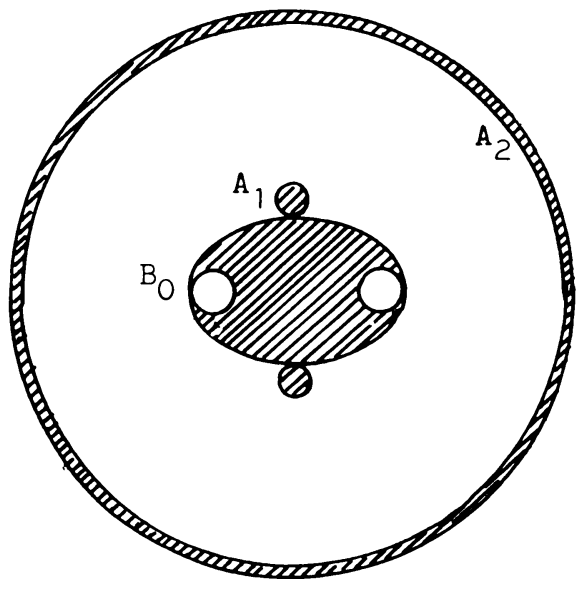

FigURE 1 
Take $\alpha>0$ small so that $\psi \geqslant \frac{1}{3} \Omega r^{2}$ on $A_{2}$. Note $\left.\psi\right|_{\partial S}=0$. One has $\int_{A_{1}} \psi=o(\varepsilon)$ and $\int_{B_{0}} \psi=o(\varepsilon)$. Thus

$$
\begin{aligned}
\left\langle\omega-\omega_{e}, G \omega_{e}\right\rangle & =\left\langle\omega-\omega_{e}, \psi\right\rangle \\
& =\int_{A_{2}} \psi+\int_{A_{1}} \psi-\int_{B_{0}} \psi=\int_{A_{2}} \psi+o(\varepsilon) \\
& \geqslant \frac{\Omega}{3} \int_{A_{2}} r^{2}+o(\varepsilon)=\frac{1}{3} \Omega R^{2} \varepsilon+o(\varepsilon)>0
\end{aligned}
$$

for $\varepsilon>0$ sufficient small. Then from (5.15), one has (5.14).

\section{REFERENCES}

1. V. I. Arnold, Conditions for nonlinear stability of stationary plane curlinear flows of an ideal fluid, Soviet Math. Dokl. 6 (1965), 773-777.

2. On an a priori estimate in the theory of hydrodynamical stability, Amer. Math. Soc. Transl. 79 (1969), 267-269.

3. __ Mathematical methods of classical mechanics, Graduate Texts in Math. \#60, Springer, New York, 1978.

4. T. B. Benjamin, The alliance of practical and analytic insights into the nonlinear problems of fluid mechanics, Applications of Methods of Functional Analysis to Problems of Mechanics, Lecture Notes in Math., vol. 503, Springer-Verlag, 1976, pp. 8-29.

5. J. Burbea, Vortex motion and their stability, Proc. Nonlinear Phenomena in Math. Sci. (Arlington), Academic Press, 1982, pp. 147-158.

6. G. S. Deem and N. J. Zabusky, Vortex waves: stationary 'V-states', interactions, recurrence, and breaking, Phys. Rev. Lett. 40 (1978), 859-862.

7. D. G. Dritschel, The stability and energetics of co-rotating uniform vortics (preprint), 1984.

8. E. Ebin and J. Marsden, Groups of diffeomorphisms and the motion of an incompressible fluid, Ann. of Math. 92 (1970), 102-163.

9. L. Kelvin (Sir W. Thomson), On the vibrations of a columnar vortex, Philos. Mag. 5 (1880), 155.

10. H. Lamb, Hydrodynamics, Dover, New York, 1945.

11. A. E. H. Love, On the stability of certain vortex motions, Proc. Roy. Soc. London 25 (1893), 18-42.

12. J. Marsden and A. Weinstein, Coadjoint orbits, vortices, and Clebsch variables for incompressible fluids, Phys. D 7 (1983), 305-323.

13. T. G. McKee, Existence and structure on non-circular stationary vortices, Thesis, Brown University, 1981.

14. R. T. Pierrehumbert, A family of steady, translating vortex pairs with distributed vorticity, J. Fluid Mech. 99 (1980), 129-144.

15. P. G. Saffman, Vortex interactions and coherent structures in turbulence, Transition and Turbulence (Ed., R. E. Meyer), Academic Press, 1981, pp. 149-166.

16. B. Turkington, On steady vortex flow in two dimensions. I, Comm. Partial Differential Equations 8 (1983), 999-1030.

17. On the evolution of a concentrated vortex in an ideal fluid (preprint), 1984.

18. Y.-H. Wan and M. Pulvirenti, Nonlinear stability of circular vortex patches, Comm. Math. Phys. 99 (1985), 435-450.

Department of Applied Mathematics, Beijing Polytechnic University, Beijing, P. R. China 